ARTICLE

\title{
Single-photon quantum regime of artificial radiation pressure on a surface acoustic wave resonator
}

\author{
Atsushi Noguchi (10) ${ }^{1,2,3 凶}$, Rekishu Yamazaki ${ }^{1}$, Yutaka Tabuchi (10 ${ }^{1}$ \& Yasunobu Nakamura (i) ${ }^{1,4}$
}

Electromagnetic fields carry momentum, which upon reflection on matter gives rise to the radiation pressure of photons. The radiation pressure has recently been utilized in cavity optomechanics for controlling mechanical motions of macroscopic objects at the quantum limit. However, because of the weakness of the interaction, attempts so far had to use a strong coherent drive to reach the quantum limit. Therefore, the single-photon quantum regime, where even the presence of a totally off-resonant single photon alters the quantum state of the mechanical mode significantly, is one of the next milestones in cavity optomechanics. Here we demonstrate an artificial realization of the radiation pressure of microwave photons acting on phonons in a surface acoustic wave resonator. The order-ofmagnitude enhancement of the interaction strength originates in the well-tailored, strong, second-order nonlinearity of a superconducting Josephson junction circuit. The synthetic radiation pressure interaction adds a key element to the quantum optomechanical toolbox and can be applied to quantum information interfaces between electromagnetic and mechanical degrees of freedom.

\footnotetext{
${ }^{1}$ Research Center for Advanced Science and Technology (RCAST), The University of Tokyo, Meguro-ku, Tokyo 153-8904, Japan. ${ }^{2}$ PRESTO, Japan Science and Technology Agency, Kawaguchi-shi, Saitama 332-0012, Japan. ${ }^{3}$ Komaba Institute for Science (KIS), The University of Tokyo, Meguro-ku, Tokyo 153-8902, Japan. ${ }^{4}$ Center for Emergent Matter Science (CEMS), RIKEN, Wako-shi, Saitama 351-0198, Japan. ${ }^{凶}$ email: u-atsushi@g.ecc.u-tokyo.ac.jp
} 
T he radiation pressure of electromagnetic field ${ }^{1}$ is one of the fundamental concepts in cavity optomechanics ${ }^{2}$. Even though the interaction is rather weak at the single-photon level, one can apply a strong drive field to enhance the effective coupling strength to reach the quantum regime ${ }^{3-8}$. Based on this interaction, ground-state cooling and quantum state control of mechanical oscillators have been reported on suspended membranes $^{3-5}$, phononic-crystal cavities ${ }^{6-8}$, micro-toroidal resonators ${ }^{9}$, and bulk oscillators ${ }^{10}$. For such experiments, however, strong drive fields often restrict quantum-limited functionalities of the optomechanical systems by introducing noise, heat and other dissipations.

The single-photon quantum regime is reached when the radiation pressure of a single photon is strong enough to overcome other dissipations in the system ${ }^{2}$, where the quantum state of the mechanical mode is coherently controlled by the quantum of the electromagnetic field. However, such a strong radiation pressure interaction has been elusive in optomechanical systems studied so far, while there are a few experiments approaching this regime $^{11,12}$.

Here we introduce an artificial optomechanical system consisting of a surface acoustic wave (SAW) resonator and a superconducting circuit. The conventional radiation pressure arises from the frequency shift of the optical (or electrical) resonator depending on the displacement of the mechanical system (Fig. 1a). Instead, we utilize a superconducting circuit with Josephson junctions, which are known to be a versatile platform for engineering strong nonlinearity with negligible dissipation ${ }^{13-16}$. The current induced by the acoustic waves in a piezoelectric material modulates the inductive energy of the Josephson circuit, which results in the motion-dependent frequency shift of the electrical resonator. The enhanced artificial radiation pressure enables us to reach the single-photon quantum regime.

\section{Results}

System and the model. Our hybrid system is composed of a Fabry-Pérot-type SAW resonator defined by a pair of Bragg mirrors $^{17}$, and a nonlinear microwave (MW) resonator (Fig. 1). They are coupled to each other via an interdigitated transducer (IDT) through the piezoelectric interaction. All the structures are made of aluminum evaporated on a ST-X quartz substrate (see the details in the "Method" section and Supplementary Note 1).

The nonlinear MW resonator consists of a short coplanar waveguide connected to the IDT on one end. On the other end it is grounded via a loop interrupted by one small and two large Josephson junctions with the Josephson energies $E^{\prime}$ and $E_{\mathrm{J}}$, a
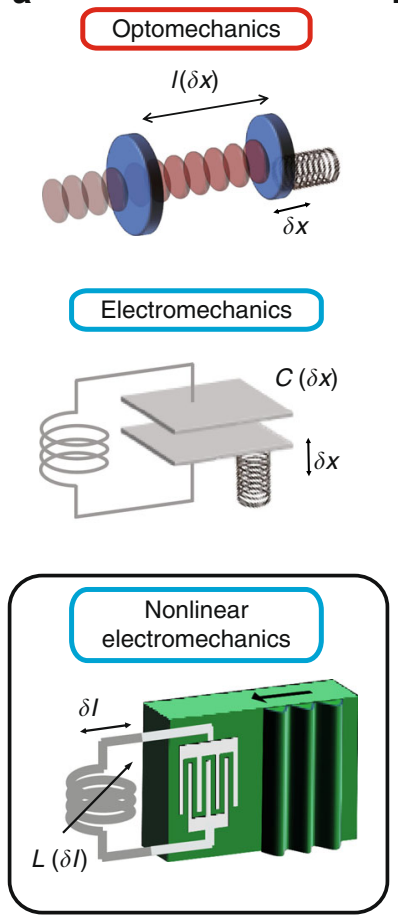

b

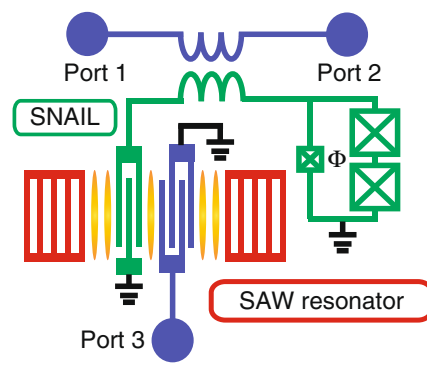

f

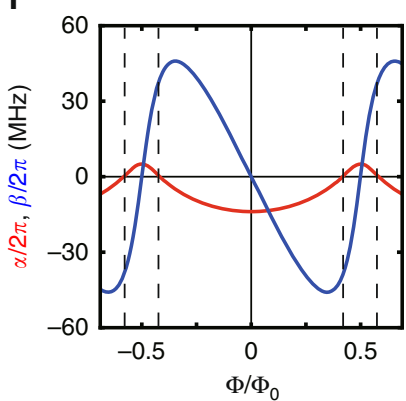

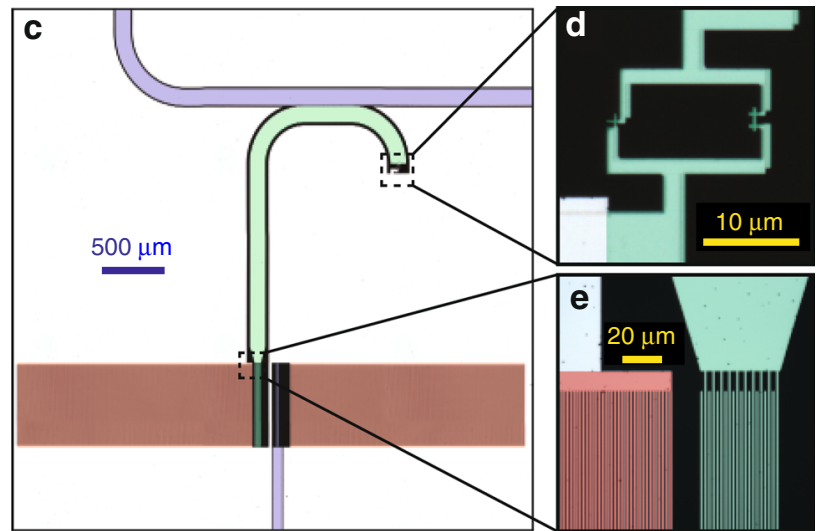

g

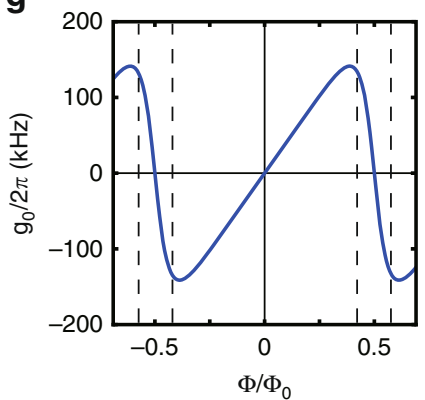

$\mathbf{h}$

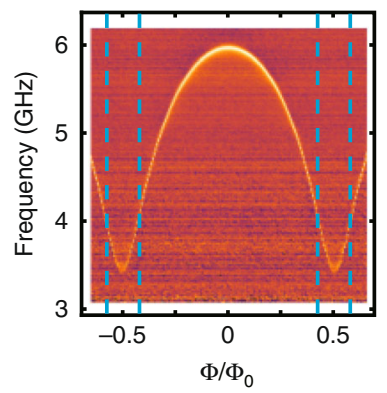

Fig. 1 SAW-MW hybrid system for artificial radiation pressure interaction. a Variety of optomechanical systems. (top) Optomechanical system using an optical cavity. The effective cavity length $/$ is modulated by the displacement of the mechanical oscillator $\delta x$. (middle) Electromechanics using a microwave lumped-element circuit. The capacitance $C$ is modulated by the displacement of the mechanical oscillator. (bottom) Electromechanics with a nonlinear Josephson junction circuit and a SAW resonator. The inductance $L$ is modulated by the current induced by transduction from acoustic waves. $\mathbf{b}$ Schematic of the SAW optomechanical system. A SAW resonator defined by Bragg mirrors (red) couples to a nonlinear MW resonator (green) via an interdigitated transducer. A SNAIL loop consisting of three Josephson junctions works as a nonlinear inductive element. Ports 1 and 2 are external feed lines for the MW resonator, and port 3 is that for the SAW resonator having a spatial mode shown in yellow. c-e False-colored micrographs of the sample. The colors of the electrodes correspond to the ones in the schematic in $\mathbf{b}$. $\mathbf{d}$ Magnification of the SNAIL part. The three junctions form the SNAIL loop. $\mathbf{e}$ Zoom-up of a part of the SAW resonator and the interdigitated transducer. $\mathbf{f}$ Calculated nonlinearity of the MW resonator as a function of the magnetic flux $\Phi$ penetrating through the SNAIL loop. Red (blue) curve represents the self-Kerr (Pockels) nonlinearity $\alpha(\beta)$ of the MW resonator. $\mathbf{g}$ Calculated strength $g_{0}$ of the artificial radiation pressure interaction induced by the nonlinearity of the SNAIL. $\mathbf{h}$ Spectrum of the nonlinear resonator as a function of $\Phi$ measured with a weak MW probe whose average intra-resonator photon number is much less than unity. Vertical dashed lines in $\mathbf{f}-\mathbf{h}$ indicate flux bias conditions where the self-Kerr nonlinearity vanishes in the numerical simulation. 
respectively. The circuit element is called the Superconducting Nonlinear Asymmetric Inductive eLement (SNAIL) ${ }^{18}$. The SNAIL has the inductive energy

$$
U(\theta)=-E_{\mathrm{J}}^{\prime} \cos \theta-2 E_{\mathrm{J}} \cos \left(\frac{\phi-\theta}{2}\right),
$$

where $\theta$ is the superconducting phase across the small junction, $\phi=2 \pi \Phi / \Phi_{0}$ is the reduced magnetic flux, $\Phi$ is the flux threading the loop, and $\Phi_{0}=h / 2 e$ is the flux quantum. The SNAIL capacitively shunted with the coplanar waveguide forms the nonlinear MW resonator, whose Hamiltonian reads (with $\hbar=1$ )

$$
\hat{H}_{\mathrm{m}}=\omega_{\mathrm{m}} \hat{a}^{\dagger} \hat{a}+\alpha_{0} \hat{a}^{\dagger} \hat{a}^{\dagger} \hat{a} \hat{a}+\beta\left(\hat{a}^{\dagger} \hat{a}^{\dagger} \hat{a}+\text { h.c. }\right) .
$$

Here $\hat{a}\left(\hat{a}^{\dagger}\right)$ is the annihilation (creation) operator of a photon in the MW resonator, and $\omega_{\mathrm{m}}$ is the resonance frequency. The terms with coefficients $\alpha_{0}$ and $\beta$ represent the nonlinearities of the resonator corresponding to the self-Kerr and Pockels effects, respectively (see the details in Supplementary Note 2).

The Hamiltonian of the hybrid system consisting of the MW and SAW resonators is written as

$$
\hat{H}=\hat{H}_{0}+\hat{V},
$$

where

$$
\hat{H}_{0}=\omega_{\mathrm{m}} \hat{a}^{\dagger} \hat{a}+\omega_{\mathrm{s}} \hat{b}^{\dagger} \hat{b}
$$

and

$$
\hat{V}=\alpha_{0} \hat{a}^{\dagger} \hat{a}^{\dagger} \hat{a} \hat{a}+\beta\left(\hat{a}^{\dagger} \hat{a}^{\dagger} \hat{a}+\text { h.c. }\right)+g_{\mathrm{p}}\left(\hat{a}^{\dagger} \hat{b}+\hat{a} \hat{b}^{\dagger}\right) .
$$

Here $\hat{b}\left(\hat{b}^{\dagger}\right)$ is the annihilation (creation) operator of a phonon in the SAW resonator, $\omega_{\mathrm{s}}$ is the resonance frequency, and $g_{\mathrm{p}}$ is the piezoelectric coupling strength between the SAW and MW resonators. By treating $\hat{V}$ as a perturbation (see the details in Supplementary Note 3), we obtain an effective interaction Hamiltonian

$$
\begin{aligned}
\hat{V}_{\text {eff }} & =\left(\alpha_{0}-\frac{3 \beta^{2}}{\omega_{\mathrm{m}}}\right) \hat{a}^{\dagger} \hat{a}^{\dagger} \hat{a} \hat{a}-\left(\frac{2 g_{\mathrm{p}} \beta}{\omega_{\mathrm{m}}-\omega_{\mathrm{s}}}\right) \hat{a}^{\dagger} \hat{a}\left(\hat{b}^{\dagger}+\hat{b}\right), \\
& \equiv \alpha \hat{a}^{\dagger} \hat{a}^{\dagger} \hat{a} \hat{a}+g_{0} \hat{a}^{\dagger} \hat{a}\left(\hat{b}^{\dagger}+\hat{b}\right),
\end{aligned}
$$

under the rotating-wave approximation. This derivation is valid when $\left\{\omega_{\mathrm{m}}, \omega_{\mathrm{s}}, \omega_{\mathrm{m}}-\omega_{\mathrm{s}}\right\} \gg\left\{\left|\alpha_{0}\right|,|\beta|,\left|g_{\mathrm{p}}\right|\right\}$ and $\omega_{\mathrm{m}} \gg \omega_{\mathrm{s}}$ are satisfied. The second term on the right-hand side represents an artificial radiation pressure interaction analogous to the Pockels effect. On the other hand, the undesired first term corresponds to a self-Kerr nonlinearity, which can be eliminated by finding experimental conditions where $\alpha$ vanishes. As we will show later, this condition mitigates the saturation effect and provides full functionality of the realized artificial radiation pressure.

Figure if shows the calculated strengths of the self-Kerr nonlinearity $\alpha$ and Pockels nonlinearity $\beta$ for the parameters of our sample. Notably, $\alpha$ vanishes at certain flux bias conditions $\Phi=\left\{\Phi_{\alpha=0}, \Phi_{0}-\Phi_{\alpha=0}\right\}$ mod $\Phi_{0}$ (vertical dashed lines in Fig. 1f-h),

while $\beta$ remains finite at the conditions. In canonical optomechanical systems, the resonance frequency of the optical (or electrical) resonator is directly affected by the displacement of the mechanical oscillator. Here, in contrast, the resonance frequency of the MW resonator is modulated by the current excited by the mechanical oscillations through the piezoelectric effect, resulting in the Pockels nonlinearity and the synthetic optomechanical coupling. Figure 1g shows the calculated strength $g_{0}$ of the artificial radiation pressure interaction. It is of importance that $g_{0}$ takes a large value at the flux bias condition where $\alpha$ vanishes.
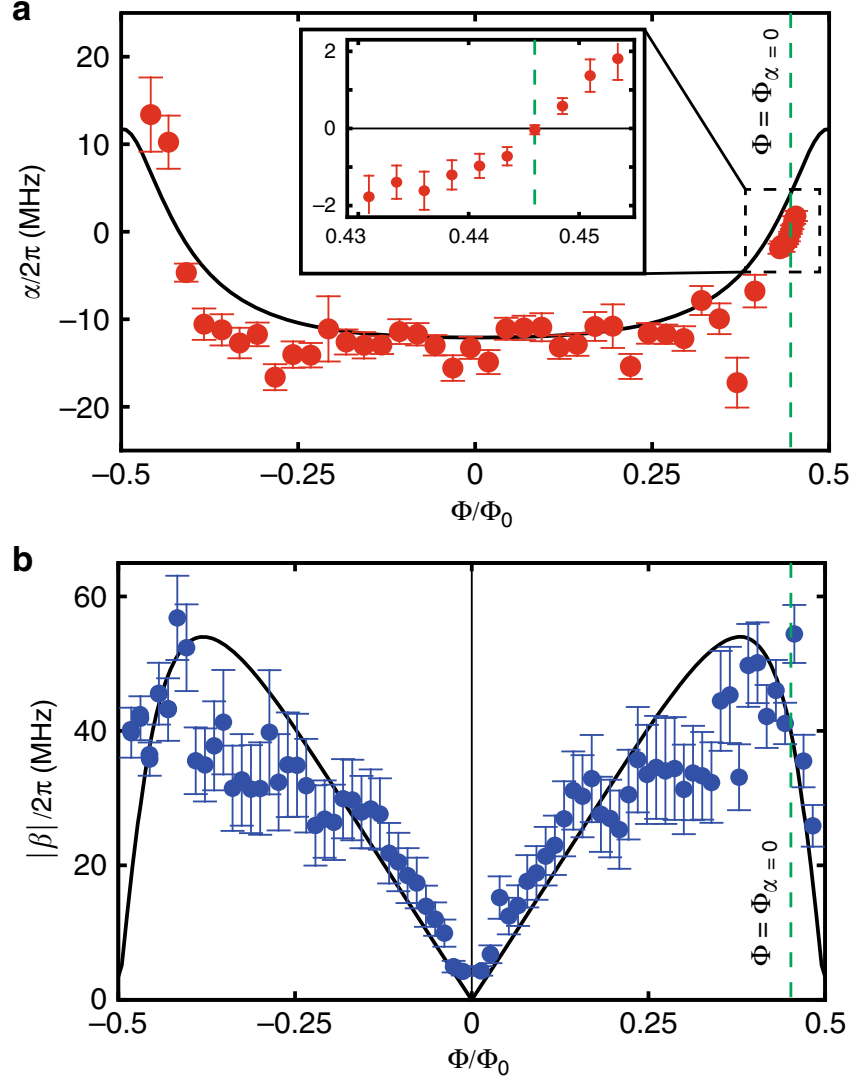

Fig. 2 Nonlinearity of the $\mathbf{M W}$ resonator. a Self-Kerr nonlinearity $\alpha$ as a function of the flux through the SNAIL loop. Inset shows an enlarged view around $\Phi=\Phi_{\alpha=0}$. b Absolute value of the Pockels nonlinearity $|\beta|$, obtained from the phonon-to-photon conversion experiment, as a function of the magnetic flux bias. The curve in each panel shows the result of the numerical simulation without any fitting parameters. Green dashed lines indicate $\Phi=0.445 \Phi_{0} \equiv \Phi_{\alpha=0}$. The theoretical curves are the same as those in Fig. 1f. Error bars represent standard errors.

Nonlinearity of the circuit. Figure 2a shows the experimentally determined self-Kerr nonlinearity $\alpha$ as a function of the flux bias. For that, we measure the shift of the MW resonator frequency per average intra-resonator probe photon number as a function of the flux bias. The observed self-Kerr nonlinearity changes its sign near $\Phi= \pm 0.5 \Phi_{0}$, as expected. The relatively large scattering of the experimental data points are presumably due to the uncertainty in the determination of the probe photon number in the resonator because of the strongly flux-dependent loss rates of the MW resonator (see Supplementary Figure 1). As shown in the inset, $\alpha$ vanishes at $\Phi_{\alpha=0} \equiv 0.445 \Phi_{0}$. At this bias point, the MW resonator frequency is $\omega_{\mathrm{m}} / 2 \pi=3.85 \mathrm{GHz}$ (Fig. 1h), largely detuned from the SAW resonator frequency $\omega_{\mathrm{s}} / 2 \pi=785.25 \mathrm{MHz}$.

To evaluate the strength of Pockels nonlinearity $\beta$, we perform a phonon-to-photon conversion experiment from the SAW resonator to the MW resonator. We irradiate the MW resonator with the red-sideband drive at frequency $\omega_{\mathrm{d}} \sim \omega_{\mathrm{m}}-\omega_{\mathrm{s}}$. Under the rotating wave approximation in the resolved-sideband limit, i.e., $\kappa \ll \omega_{\text {s }}$, where $\kappa$ is the total decay of the MW resonator, the Hamiltonian becomes

$$
\hat{H}=\Delta \hat{a}^{\dagger} \hat{a}+g_{0} \sqrt{n_{\mathrm{d}}}\left(\hat{a}^{\dagger} \hat{b}+\hat{a} \hat{b}^{\dagger}\right),
$$

where $n_{\mathrm{d}}$ is the average photon number of the drive field in the MW resonator and $\Delta \equiv \omega_{\mathrm{m}}-\omega_{\mathrm{d}}-\omega_{\mathrm{s}}$ is the detuning. The 
details of the derivation are presented in Supplementary Note 4. When the drive field is tuned to the red-sideband transition, i.e., $\Delta=0$, the two resonators are parametrically coupled to each other. Then, the excitation of the SAW resonator is converted to the excitation of the MW resonator, and its output MW power $P_{\text {out }}$ can be written as

$$
P_{\text {out }}=\hbar \omega_{\mathrm{m}} \kappa_{\mathrm{ex}} n_{\mathrm{s}} \frac{4 C_{0} n_{\mathrm{d}}}{\left(1+C_{0} n_{\mathrm{d}}\right)^{2}},
$$

where $C_{0} \equiv 4 g_{0}^{2} /(\kappa \Gamma)$ is the single-photon cooperativity between the SAW and MW resonators, $\kappa$ and $\Gamma$ are the respective total loss rates, and $\kappa_{\mathrm{ex}}$ is the external coupling of the MW resonator. The average intra-resonator photon number $n_{\mathrm{d}}$ of the drive field and the intra-resonator phonon number $n_{\mathrm{s}}$ of the SAW resonator are calibrated by the saturation effect and the Stark shift of the MW resonator, respectively (see Supplementary Figs. 4 and 5). Here, for the phonon-to-photon conversion, we use a weak drive field which provides a small $n_{\mathrm{d}}(\sim 0.01)$ to avoid saturating the MW resonator. Therefore, we can evaluate $C_{0}$ from Eq. (8). Acccording to the definition of $g_{0}$ in Eq. (6), the relation between $C_{0}$ and $\beta$ follows:

$$
C_{0}=\frac{16 g_{\mathrm{p}}^{2} \beta^{2}}{\kappa \Gamma\left(\omega_{\mathrm{m}}-\omega_{\mathrm{s}}\right)^{2}},
$$

from which we evaluate the strength of the Pockels nonlinearity $\beta\left[=\left(\sqrt{C_{0} \kappa \Gamma} / 4 g\right)\left(\omega_{\mathrm{m}}-\omega_{\mathrm{s}}\right)\right]$ shown in Fig. 2b. The overall behavior agrees well with the theoretical prediction.

Single photon quantum regime. We apply a stronger redsideband drive to obtain a larger cooperativity with the artificial radiation pressure. This results in the increase of the effective decay rate of the SAW resonator through the optomechanical damping. Figure $3 \mathrm{a}$ shows the spectra of the SAW resonator in the presence of the optomechanical damping rate $\Gamma_{\text {opt }}$. The total linewidth $\Gamma_{\text {all }}$ of the spectrum is given by

$$
\Gamma_{\text {all }}=\Gamma+\Gamma_{\text {opt }}=(1+C) \Gamma,
$$

from which we evaluate the cooperativity $C$.

Figure $3 \mathrm{~b}$ shows the cooperativity $C$ as a function of the drive photon number $n_{\mathrm{d}}$ at $\Phi=0.445 \Phi_{0}=\Phi_{\alpha=0}$ and $\Phi=0.264 \Phi_{0}$, respectively. Because of the absence of the self-Kerr nonlinearity, the saturation effect is much less pronounced at $\Phi=\Phi_{\alpha=0}$, allowing us to drive the system, as in other conventional optomechanical systems, to enhance the radiation pressure interaction. The cooperativity reaches 5 at high drive power. The remaining saturation effect is presumably due to the higherorder nonlinearities beyond the third order. The slope of the cooperativity for the small drive photon number corresponds to the single-photon cooperativity $C_{0}$, which is determined to be $1.7 \pm 0.1$ from the linear fit in Fig. 3. Thus, the singlephoton quantum regime $C_{0}>1$ is achieved here. From this value, the optomechanical coupling strength is evaluated to be $g_{0} / 2 \pi=190 \mathrm{kHz}$, which agrees well with the calculation shown in Fig. 1g. It is also consistent with the estimation from the peak power of the up-conversion signal [Eq. (9)], which gives $g_{0} / 2 \pi=230 \mathrm{kHz}$.

\section{Discussions}

Having established the single-photon quantum regime, we compare various realizations of optomechanical systems $3,6,9,12,19-40$ from the viewpoint of quantum-limited measurement of phonons. Figure 4 shows the minimum intra-resonator photon number $N_{\mathrm{q}}=\left(n_{\mathrm{th}}+1 / 2\right) / C_{0}$, whose back-action shot noise on the mechanical mode becomes dominant over the thermal and
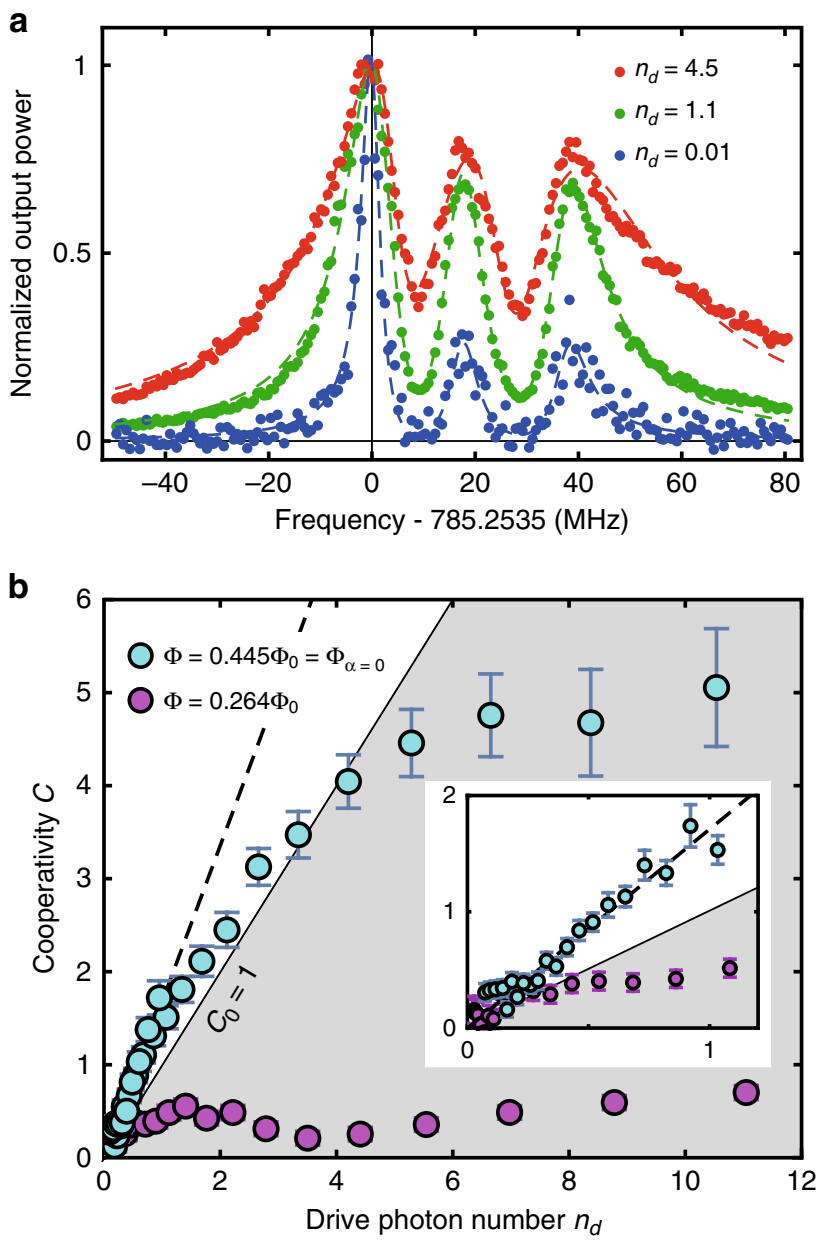

Fig. 3 Strong artificial radiation pressure. a Normalized output power of the MW signal coherently up-converted from the SAW excitations as a function of the detuning of the SAW drive frequency (dots). Three datasets are for different drive powers represented by the average number, $n_{d}$, of drive photons in the $\mathrm{MW}$ resonator. Dashed curves are the results of fittings with a sum of three Lorentzians in the complex plane. The left-most peak is from the fundamental transverse mode of the SAW resonator, while the two other peaks are due to higher-index transverse modes. b Cooperativity $C$ as a function of the drive photon number $n_{\mathrm{d}}$. White area corresponds to the single-photon quantum regime, i.e., $C_{0}>1$. Cyan dots are the experimental data at $\Phi=0.445 \Phi_{0}=\Phi_{\alpha=0}$. Purple dots are taken at $\Phi=0.264 \Phi_{0}$, where $\alpha \neq 0$ and the saturation takes place at lower power. Black dashed line is the linear fit for the cyan dots in the low-power region. Inset shows an enlarged view near the origin. Error bars represent standard errors.

vacuum noises ${ }^{2}$. Here $n_{\text {th }}=1 /\left(\mathrm{e}^{\hbar \omega_{\text {mech }} / k_{\mathrm{B}} T}-1\right), \omega_{\text {mech }}$ is the mechanical mode frequency, $T$ is the bath temperature, and $k_{\mathrm{B}}$ is Boltzmann constant. The experiment was conducted at $T=40$ $\mathrm{mK}$, which gives $N_{\mathrm{q}}$ of $0.67 \pm 0.04 . N_{\mathrm{q}}$ becomes less than unity when the kick by a single intra-resonator photon is larger than the mechanical oscillator beyond its noise amplitude of the motion. In this regime, the quantum-limited quadrature measurement of the mechanical oscillator and the mechanically induced transparency of the MW resonator can be realized with single intra-resonator photons. The current device is in the resolved-sideband limit, however, such that the incident drive is strongly filtered by the resonator and needs to be a large amplitude even if the required intra-resonator photon number is less than unity. To solve the problem, we can use a triple resonance technique ${ }^{41}$ to improve the 


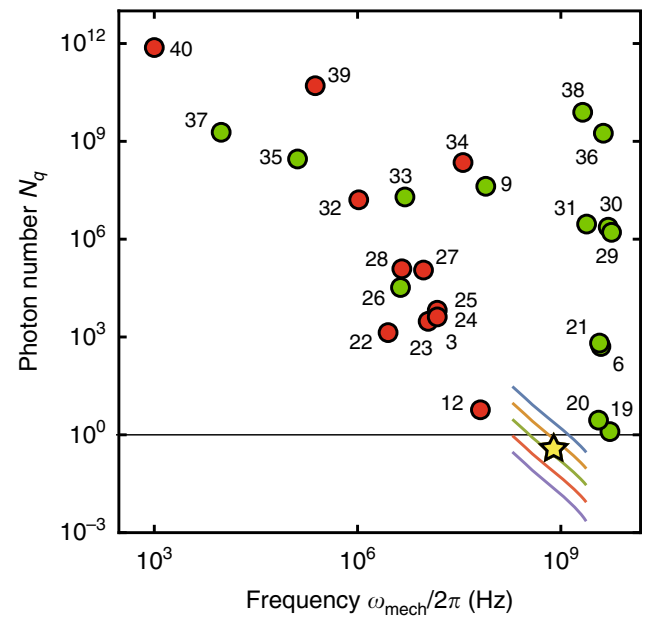

Fig. 4 Single-photon quantum regime. Each data point shows intraresonator drive photon number $N_{\mathrm{q}}$ necessary for the quantum-limited quadrature measurement of phonons as a function of mechanical eigenmode frequency $\omega_{\text {mech }} / 2 \pi$. Green (red) circles indicate the values found in other opto-(electro-)mechanical systems with radiation pressure interaction. The yellow star shows the value obtained in this work. Color curves show the expectations from numerical simulations. Different colors correspond to the values of $E_{J} / E_{C}=\left\{10^{5}, 10^{4}, 10^{3}, 10^{2}, 10\right\}$ from top to bottom. $A$ list of the references indicated by the numbers next to the circles is presented in the reference list.

coupling efficiency of the incident drive and utilize a quantum feature of the drive field.

The single-photon quantum regime is also useful for the quantum control of the mechanical oscillator through the radiation pressure interaction. When $N_{\mathrm{q}}$ is less than unity, we can safely use a two-level system, i.e., a qubit, instead of a nearly harmonic microwave resonator. Then, quantum control of the SAW resonator can be readily demonstrated. Recently, quanta of acoustic waves are being controlled and monitored by using a resonantly ${ }^{42,43}$ or dispersively 44,45 coupled superconducting qubit. In contrast to those demonstrations, the radiation pressure interaction between a SAW resonator and a superconducting qubit gives rise to a 'spin' dependent force, which can be used, e.g., for generating a Schödinger's cat state of a SAW resonator, as recently demonstrated in a vibrational mode in a trapped-ion experiment ${ }^{46}$. The spin-dependent force also enables fast entangling gates in analogy with those in trapped-ion systems ${ }^{47,48}$.

Moreover, as the numerical simulations indicate (color lines in Fig. 4), we can readily increase the coupling strength further by using a SAW resonator with higher frequency to reduce the detuning from the MW resonator. The condition $g_{0} / \kappa>1$ is also within the scope of the future experiment, where the presence of a single phonon shifts the resonance frequency of the MW resonator by more than its linewidth ${ }^{49}$. It will then allow for observation of quantum jumps between phonon Fock states ${ }^{50}$.

\section{Method}

Sample parameters. The parameters in the sample are the following. At zero flux bias, the resonance frequency of the MW resonator is $\omega_{\mathrm{m}} / 2 \pi=5.98 \mathrm{GHz}$, and the internal and total loss rates are 10 and $55 \mathrm{MHz}$, respectively. At $\Phi=\Phi_{\alpha=0} \equiv$ $0.445 \Phi_{0}$, the resonance frequency is $3.85 \mathrm{GHz}$, the internal loss rate $\kappa_{\mathrm{in}} / 2 \pi=3$ $\mathrm{MHz}$, and the external loss rate $\kappa_{\mathrm{ex}} / 2 \pi=17 \mathrm{MHz}$. The resonance frequency of the SAW resonator is found to be $\omega_{\mathrm{s}} / 2 \pi=785.25 \mathrm{MHz}$, together with the total loss rate $\Gamma / 2 \pi=4.4 \mathrm{kHz}$. The external coupling rate is designed to be $\Gamma_{\text {ex }} / 2 \pi=0.6 \mathrm{kHz}$. The total loss rate of the MW resonator $\kappa / 2 \pi=20 \mathrm{MHz}$ is much smaller than the resonance frequency of the SAW resonator. The piezoelectric coupling strength evaluated from the frequency shift of the SAW resonator under a strong drive is $g_{\mathrm{p}} / 2 \pi=6.4 \mathrm{MHz}^{17}$. The strength of the self-Kerr nonlinearity at zero flux bias is determined as $\alpha_{0} / 2 \pi=-13.0 \mathrm{MHz}$ (see the details in Supplementary Note 5). The bath temperature in the experiment was at $T=40 \mathrm{mK}$.

\section{Data availability}

The data that support the findings of this study are available from the corresponding author upon reasonable request.

Received: 25 November 2019; Accepted: 10 February 2020;

Published online: 17 March 2020

\section{References}

1. Nichols, E. F. \& Hull, G. F. A preliminary communication on the pressure of heat and light radiation. Phys. Rev. 13, 307-320 (1901).

2. Aspelmeyer, M., Kippenberg, T. J. \& Marquardt, F. Cavity optomechanics. Rev. Mod. Phys. 86, 1391-1452 (2014).

3. Teufel, J. D. et al. Sideband cooling of micromechanical motion to the quantum ground state. Nature 475, 359-363 (2011).

4. Lecocq, F., Teufel, J. D., Aumentado, J. \& Simmonds, R. W. Resolving the vacuum fluctuations of an optomechanical system using an artificial atom. Nat. Phys. 11, 635-639 (2015).

5. Noguchi, A. et al. Ground state cooling of a quantum electromechanical system with a silicon nitride membrane in a 3D loop-gap cavity. New J. Phys 18, 103036 (2016)

6. Chan, J. et al. Laser cooling of a nanomechanical oscillator into its quantum ground state. Nature 478, 89-92 (2011).

7. Hong, S. et al. Hanbury Brown and Twiss interferometry of single phonons from an optomechanical resonator. Science 358, 203-206 (2017).

8. Purdy, T. P., Grutter, K. E., Srinivasan, K. \& Taylor, J. M. Quantum correlations from a room-temperature optomechanical cavity. Science 356, 1265-1268 (2017).

9. Verhagen, E., Deléglise, S., Weis, S., Schliesser, A. \& Kippenberg, T. J. Quantum-coherent coupling of a mechanical oscillator to an optical cavity mode. Nature 482, 63-67 (2012).

10. Santos, J. T., Li, J., Ilves, J., Ockeloen-Korppi, C. F. \& Sillanpää, M. Optomechanical measurement of a millimeter-sized mechanical oscillator approaching the quantum ground state. New J. Phys. 19, 103014 (2017).

11. Meenehan, S. M. et al. Silicon optomechanical crystal resonator at millikelvin temperatures. Phys. Rev. A 90, 011803(R) (2014).

12. Pirkkalainen, J.-M. et al. Cavity optomechanics mediated by a quantum twolevel system. Nat. Commun. 6, 6981 (2015).

13. Nakamura, Y., Pashkin, Y. A. \& Tsai, J. S. Coherent control of macroscopic quantum states in a single-Cooper-pair box. Nature 398, 786-788 (1999)

14. Wallraff, A. et al. Strong coupling of a single photon to a superconducting qubit using circuit quantum electrodynamics. Nature 431, 162-167 (2004).

15. Yamamoto, T. et al. Flux-driven Josephson parametric amplifier. Appl. Phys. Lett. 93, 042510 (2008)

16. Roy, A. \& Devoret, M. H. Introduction to quantum-limited parametric amplification of quantum signals with Josephson circuits. C. R. Phys. 17, 740-755 (2016)

17. Noguchi, A., Yamazaki, R., Tabuchi, Y. \& Nakamura, Y. Qubit-assisted transduction for a detection of surface acoustic waves near the quantum limit Phys. Rev. Lett. 119, 180505 (2017).

18. Frattini, N. E. et al. 3-wave mixing Josephson dipole element. Appl. Phys. Lett 110, 222603 (2017)

19. Riedinger, R. et al. Non-classical correlations between single photons and phonons from a mechanical oscillator. Nature 530, 313-316 (2016).

20. Meenehan, S. M. et al. Silicon optomechanical crystal resonator at millikelvin temperatures. Phys. Rev. A 90, 011803(R) (2014).

21. Krause, A. G. et al. Nonlinear radiation pressure dynamics in an optomechanical crystal. Phys. Rev. Lett. 115, 233601 (2015).

22. Pirkkalainen, J.-M., Damskägg, E., Brandt, M., Massel, F. \& Sillanpää, M. A. Squeezing of quantum noise of motion in a micromechanical resonator. Phys. Rev. Lett. 115, 243601 (2015).

23. Barzanjeh, S. et al. Stationary entangled radiation from micromechanical motion. Nature 570, 480-483 (2019).

24. Lecocq, F., Clark, J. B., Simmonds, R. W., Aumentado, J. \& Teufel, J. D. Mechanically mediated microwave frequency conversion in the quantum regime. Phys. Rev. Lett. 116, 043601 (2016).

25. Lecocq, F., Clark, J. B., Simmonds, R. W., Aumentado, J. \& Teufel, J. D. Quantum nondemolition measurement of a nonclassical state of a massive object. Phys. Rev. X 5, 041037 (2015).

26. Wilson, D. J. et al. Measurement-based control of a mechanical oscillator at its thermal decoherence rate. Nature 524, 325-329 (2015). 
27. Teufel, J. D., Lecocq, F. \& Simmonds, R. W. Overwhelming thermomechanical motion with microwave radiation pressure shot noise. Phys. Rev. Lett. 116, 013602 (2016).

28. Fink, J. M. et al. Quantum electromechanics on silicon nitride nanomembranes. Nat. Commun. 7, 12396 (2016).

29. Cohen, J. D. et al. Phonon counting and intensity interferometry of a nanomechanical resonator. Nature 520, 522-525 (2015).

30. Fang, K. et al. Generalized non-reciprocity in an optomechanical circuit via synthetic magnetism and reservoir engineering. Nat. Phys. 13, 465-471 (2017).

31. Balram, K. C., Davanco, M. I., Song, J. D. \& Srinivasan, K. Coherent coupling between radiofrequency, optical and acoustic waves in piezo-optomechanical circuits. Nat. Phys. 10, 346-352 (2016).

32. Teufel, J. D., Donner, T., Castellanos-Beltran, M. A., Harlow, J. W. \& Lehnert, K. W. Nanomechanical motion measured with an imprecision below that at the standard quantum limit. Nat. Nano 4, 820-823 (2009).

33. Shkarin, A. B. et al. Optically mediated hybridization between two mechanical modes. Phys. Rev. Lett. 112, 013602 (2014).

34. Singh, V. et al. Optomechanical coupling between a multilayer graphene mechanical resonator and a superconducting microwave cavity. Nat. Nano $\mathbf{9}$, 820-824 (2014).

35. Thompson, J. D. et al. Strong dispersive coupling of a high-finesse cavity to a micromechanical membrane. Nature 452, 75 (2008).

36. Bochmann, J., Vainsencher, A., Awschalom, D. D. \& Cleland, A. N Nanomechanical coupling between microwave and optical photons. Nat. Phys. 9, 712-716 (2013).

37. Kleckner, D. et al. Optomechanical trampoline resonators. Opt. Express 19, 19708-19716 (2011).

38. Mitchell, M. et al. Single-crystal diamond low-dissipation cavity optomechanics. Optica 3, 963-970 (2016).

39. Regal, C. A., Teufel, J. D. \& Lehnert, K. W. Measuring nanomechanical motion with a microwave cavity interferometer. Nat. Phys. 4, 555-560 (2008).

40. Cuthbertson, B. D., Tobar, M. E., Ivanov, E. N. \& Blair, D. G. Parametric backaction effects in a high-Q cryogenic sapphire transducer. Rev. Sci. Instrum. 67, 2435-2442 (1996).

41. Rueda, A. et al. Efficient microwave to optical photon conversion: an electrooptical realization. Optica 3, 597-604 (2016).

42. Satzinger, K. J. et al. Quantum control of surface acoustic-wave phonons. Nature 563, 661-665 (2018).

43. Chu, Y. et al. Creation and control of multi-phonon Fock states in a bulk acoustic-wave resonator. Nature 563, 666-670 (2018).

44. Sletten, L. R., Moores, B. A., Viennot, J. J. \& Lehnert, K. W. Resolving phonon fock state in a multimode cavity with a double-slit qubit. Phys. Rev. X 9, 021056 (2019)

45. Arrangoiz-Arriola, P. et al. Resolving the energy levels of a nanomechanical oscillator. Nature 571, 537-540 (2019).

46. Kienzler, D. et al. Observation of quantum interference between separated mechanical oscillator wave packets. Phys. Rev. Lett. 116, 140402 (2016).

47. Turchette, Q. A. et al. Deterministic entanglement of two trapped ions. Phys. Rev. Lett. 81, 3631 (1998).

48. Ballance, C. J., Harty, T. P., Linke, N. M., Sepiol, M. A. \& Lucas, D. M. Highfidelity quantum logic gates using trapped-ion hyperfine qubits. Phys. Rev. Lett. 117, 060504 (2016)
49. Murch, K. W., Moore, K. L., Gupta, S. \& Stamper-Kurn, D. M. Observation of quantum-measurement backaction with an ultracold atomic gas. Nat. Phys. $\mathbf{4}$ 561-564 (2008)

50. Miao, H., Danilishin, S., Corbitt, T. \& Chen, Y. Standard quantum limit for probing mechanical energy quantization. Phys. Rev. Lett. 103, 100402 (2009).

\section{Acknowledgements}

The authors acknowledge K. Kusuyama for the help in sample fabrication. This work was partly supported by JSPS KAKENHI (Grant number 26220601), JST PRESTO (Grant number JPMJPR1429), and JST ERATO (Grant number JPMJER1601).

\section{Author contributions}

A.N. designed and fabricated the devices. A.N. also conducted the experiments and analyzed the data. Y.N. supervised the project. A.N., R.Y., Y.T., and Y.N. discussed the results and wrote the manuscript together.

\section{Competing interests}

The authors declare no competing interests.

\section{Additional information}

Supplementary information is available for this paper at https://doi.org/10.1038/s41467020-14910-z.

Correspondence and requests for materials should be addressed to A.N.

Peer review information Nature Communications thanks the anonymous reviewers for their contribution to the peer review of this work. Peer reviewer reports are available.

Reprints and permission information is available at http://www.nature.com/reprints

Publisher's note Springer Nature remains neutral with regard to jurisdictional claims in published maps and institutional affiliations.

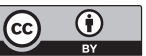

Open Access This article is licensed under a Creative Commons Attribution 4.0 International License, which permits use, sharing, adaptation, distribution and reproduction in any medium or format, as long as you give appropriate credit to the original author(s) and the source, provide a link to the Creative Commons licence, and indicate if changes were made. The images or other third party material in this article are included in the article's Creative Commons licence, unles indicated otherwise in a credit line to the material. If material is not included in the article's Creative Commons licence and your intended use is not permitted by statutory regulation or exceeds the permitted use, you will need to obtain permission directly from the copyright holder. To view a copy of this licence, visit http://creativecommons.org/ licenses/by/4.0/

(C) The Author(s) 2020 